\title{
TUMOR WIDTH ON T1-WEIGHTED MRI IMAGES OF GLIOBLASTOMA AS A PROGNOSTIC BIOMARKER: A MATHEMATICAL MODEL
}

\author{
Julián Pérez-Beteta, Juan Belmonte-Beitia \\ AND VÍ́CTOR M. PÉREZ-García*
}

\begin{abstract}
We construct a minimal macroscopic model of glioblastoma growth including necrosis to explain the recently observed correlation between MRI-observed features and tumor growth speed. A theoretical study of the modified model was carried out. In particular, we obtained an expression for the minimal wave speed of the traveling wave solutions. We also solved numerically the model using a set of realistic parameter values and used these numerical solutions to compare the model dynamics against patient's imaging and clinical data. The mathematical model provides theoretical support to the observation that tumors with broad contrast enhancing areas as observed in T1-weighted pretreatment postcontrast magnetic resonance images have worse survival than those with thinner areas.
\end{abstract}

Mathematics Subject Classification. 92B05, 92B99, 92C50, 92C55.

Received December 14, 2018. Accepted April 29, 2019.

\section{INTRODUCTION}

The computation of quantitative features from magnetic resonance images (MRIs) to develop biomarkers of utility in Oncology has attracted a lot attention in recent years, due to its potential applications. The main premise of that field, sometimes referred to as 'radiomics', is that diagnosis, prognosis or response to specific therapies could be associated with quantitative voxel-based features. This approach could provide more solid information than the more qualitative radiological and clinical data used currently in clinical practice [17, 41].

This belief has led to a global effort in correlating imaging features and clinical data for different types of cancer, including Glioblastoma (GBM), that is the most common and lethal malignant primary brain tumor. GBM patients have a very poor survival of around 15 months when the standard of care is used. MRI is the standard imaging technique used for diagnosis, treatment planning and follow-up in GBM. The different MRI sequences performed routinely provide clinicians with essential information on the tumor cellularity, vessel functionality, oedema, etc., and radiomics has intended to exploit that information to provide clinical benefits in the management of patients of that devastating disease. Indeed, many authors have investigated the use of

Keywords and phrases: Glioblastoma, mathematical oncology, virtual patients, in silico trials, imaging biomarkers.

Mathematical Oncology Laboratory (MOLAB), Departamento de Matemáticas, E. T. S. de Ingenieros Industriales and Instituto de Matemática Aplicada a la Ciencia y la Ingeniería (IMACI), Avda. Camilo José Cela 3, Universidad de Castilla-La Mancha, 13071 Ciudad Real, Spain.

* Corresponding author: victor.perezgarcia@uclm.es 

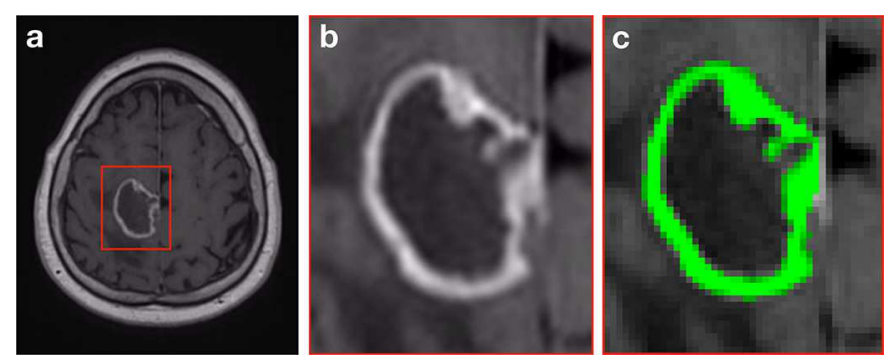

FigURE 1. T1-weigthed contrast enhanced magnetic resonance image (MRI) of a glioblastoma. (a) A two-dimensional slice of the full MRI volumetric acquisition. (b) Detail of the tumor areas showing contrast enhancement. (c) Green pixels show an example of the segmentation of contrast-enhancing areas of the tumor shown in (b).

different types of 'radiomic' quantifiers (volumetric, textural and geometrical MRI-based features) as predictors of survival or response in GBM [1, 10, 11, 13-15, 18, 19, 21, 22, 26, 29-31, 43, 44].

Glioblastoma is also one of the cancer types that has attracted more interest from the mathematical oncology community (see e.g., the reviews $[2,6,36]$ and references therein). Of the many papers available on 'mechanistic' mathematical models of glioblastoma, a few have tried to define image-based biomarkers of clinical applicability. Between them, the remarkable seminal works of Swanson et al. [40] and Wang et al. [42], based on the simplest Fisher-Kolmogorov equation that proposed the use of sequential images on T1-weighted and T2/FLAIR sequences to estimate the tumor growth speed.

Also, a metric of invasiveness related to a reaction-diffusion PDE model was proposed to find which patients could benefit the most from more aggressive resections [7]. However, the results are under question since a confirmatory work provided different results [5]. Other quantitative biomarkers to predict response to surgery based on mathematical concepts have been proposed [31].

Another work was developed on the basis that the appearance of GBM upon diagnosis, observed on contrast enhanced magnetic resonance images (MRI-T1w), consists of an enhancing area like a rim shape with central non-enhancing area corresponding to necrosis. An example is shown in Figure 1. The enhancing area is due to the signal hyper-intensity produced by the injected Gadolinium contrast, that is released in the areas of compromised blood-barrier, indicating the presence of macroscopically large amounts of tumor cells. The mathematical model developed in [35] included the necrotic tissue and led to the hypothesis that measures of the contrast-enhancing rim could provide information to construct prognostic biomarkers in GBM. A mathematical analysis of that model was developed in [3]. A first biomarker inspired by the analysis of the mathematical model was found to have a prognostic value in univariate statistics in a first limited clinical study on 117 patients with unifocal tumors. In that study, thinner widths were found to be associated with better patients prognosis [30]. Then, a larger study based on 311 patients with unifocal tumors confirmed the results and provided multivariate survival predictors based on the biomarker and other morphological and clinical data [32]. Indeed the multivariate, mathematical-model-based approach provided substantially better prognosis indicators than 'blind' machine learning approaches [27]. The biomarker was also found to be of applicability for multifocal tumors [34]. Finally, other rim-geometry-based biomarkers were also found to have a prognostic value [33].

However, the mathematical study of reference [35], although inspirational, had several limitations. The first one was that the model assumed tumor cell death to be active independently of the population of tumor cells, that is not completely realistic. The second one was that the analysis of the model involved several approximations. Although some conclusions could be intuitively guessed from the model, they have not quantitatively discussed previously.

Thus, our goal in this paper was to develop an improved (yet very simple) mathematical model describing better the biological phenomena involved in GBM growth to correlate it with imaging data. The intention of our study is not providing precise quantitative predictions of the tumor growth, but to focus only in qualitative 
properties. Specifically, we will demonstrate the principle that 'thick' tumors as observed in postcontrast T1weighted MRIs lead to worse survival that derives directly from the mathematical model.

The structure of the paper is as follows. First, in Section 2, we describe our mathematical model (Sect. 2.1), find out the appropriate parameter ranges (Sect. 2.2), define the patients data and imaging characteristics (Sect. 2.3) and other methodological questions. Next, in Section 3, we perform a theoretical study of the model. The results of the numerical simulations and the validation against patient's data are presented in Section 4 . Finally, in Section, 5 we discuss the implications of our findings and summarize our results.

\section{MODELS AND DATA}

\subsection{Mathematical model}

The mathematical model to be used in this paper is given by the equations

$$
\begin{aligned}
& \frac{\partial u}{\partial t}=D \Delta u+\rho u(1-u-v)-\left[\alpha_{1} u(u-\gamma)+\alpha_{2} u v\right] \phi(u, v), \\
& \frac{\partial v}{\partial t}=\left[\alpha_{1} u(u-\gamma)+\alpha_{2} u v\right] \phi(u, v),
\end{aligned}
$$

where

$$
\phi(u, v)=\left\{\begin{array}{lll}
1, & \text { if } & u(x, t)+v(x, t)>\gamma \\
0, & \text { if } & u(x, t)+v(x, t) \leq \gamma
\end{array}\right.
$$

with $\gamma<1$.

Equations (2.1) are a set of partial differential equations defining the growth of a tumor population $(u(x, t))$ and necrosis $(v(x, t))$ on a bounded domain $\Omega$ taken to be larger than the tumor size to avoid boundary effects.

Equation (2.1a) describes the dynamics of the tumor cells. They follow a classical Fisher-Kolmogorov equation with the addition of a tumor cell death term. This term combines two contributions, the first one is due to the damage induced to the vasculature by tumor cells. GBM cells secrete high quantities of tissue factor (TF) that induces blood coagulation inside the capillaries what results in thrombotic events. Once tumor cell density reaches the level $\gamma$, vessel damage becomes relevant and induces cell death with a rate $\alpha_{1}[24,25]$. The particular form of the term chosen ensures a gradual increase in coagulation events leading to cell death after crossing the threshold $\gamma$. The second cell loss term accounts for the contribution of necrosis as a multiplicative death term. Differently from apoptosis, or controlled cell suicide, necrosis results in the release of cell's toxic contents to the medium what induces damage to tumor cells with a rate $\alpha_{2}$.

Equation (2.1b) describes the dynamics of necrosis, whose form $\alpha_{1} u(u-\gamma)+\alpha_{2} u v$ is ruled by the death of tumor cells. For simplicity, we will take $\alpha_{1}=\alpha_{2}=\alpha$ in what follows and define $\alpha=\rho \beta$ for convenience.

Thus, our system has two different behaviors: the first one, when $u(x, t)+v(x, t) \leq \gamma$, it becomes the standard Fisher-Kolmogorov equation, whereas when $u(x, t)+v(x, t)>\gamma$ a compartment of necrotic tissue appears.

This is a simplified model that intends to describe only some basic dynamical features of GBM growth in bulk white matter. The incorporation of further details in a realistic way may require determining many parameters and biological details that are currently insufficiently understood, e.g., edema secretion, deformation of tumor and normal brain, bleeding, etc., that are beyond the purpose of our study. We did not incorporate other effects such as possible differences in speed propagation between white and grey matter. Gliomas typically arise in white matter and propagate through that medium until they reach the cortex, a moment in which they might change their speed. Grey matter is a thin layer in comparison with white matter and thus any potential changes in speed would not be too relevant for the global growth dynamics. Once the tumor reaches the grey matter it takes a short time for it to get to the meninges in comparison with the tumor evolution times. 


\subsection{Parameter estimation}

Our model equations (Eq. (2.1)) have four parameters with biological meaning. The first one is the diffusion parameter $D$ that provides an estimate of the cell motility in real brain tissue. The ranges of values for this parameter are well known, since it is a key ingredient of all models based on reaction-diffusion equations. Probably the most in depth analysis of the 'correct' value of the diffusion parameter was developed in reference [20], where a comparison between different types of models and realistic infiltration speeds was made. In line with that reference we will take $D$ to be in the range from $0.001 \mathrm{~mm}^{2}$ day ${ }^{-1}$ (low-infiltrative tumors) to $0.1 \mathrm{~mm}^{2}$ day $^{-1}$ (highly-infiltrative tumors).

For the proliferation parameter $\rho$, we will consider it to be in the range 0.01 to 0.06 day $^{-1}$, corresponding to tumor doubling times between 15 days and 2 months approximately, and in line with other works $[8,42]$.

The parameter $\gamma$ marks the threshold density above which vessel damage occurs and it is here estimated to be equal to 0.7 . For those densities a substantial fraction of the tissue is occupied by tumor cells what leads to elevated levels of tissue factor that induce thrombotic events [24, 25].

Finally, the parameter $\beta$ is less well defined and provides a death rate for the tumor tissue after vessel failures in units of the proliferation time. Here the full range $\beta \in[0.5,2.0]$ was explored what corresponds to $\alpha \in[0.005,0.12]$ day $^{-1}$, leading to characteristic death times ranging from a week to several months.

A summary of the ranges of the values tested for each parameter in this paper is shown in Table 1.

TABLE 1. Values of the parameters $D, \rho, \beta$ and $\gamma$ used in the simulations for the mathematical model (Sect. 2.1).

\begin{tabular}{ccccc}
\hline Parameter & Ranges & Steps & Units & References \\
\hline$D$ & {$[0.001-0.1]$} & 0.005 & $\mathrm{~mm}^{2}$ day $^{-1}$ & {$[8,20]$} \\
$\rho$ & {$[0.01-0.06]$} & 0.01 & day $^{-1}$ & {$[8,42]$} \\
$\beta$ & {$[0.5-2]$} & 0.5 & - & Estimated \\
$\gamma$ & 0.7 & - & - & Estimated \\
\hline
\end{tabular}

\subsection{Patients}

This study was based on data from the retrospective observational studies GLIOMAT and TOG. These studies were approved by the Institutional Review Board (IRB) of the participating local institutions. All personal data were treated anonymously and following the principles expressed in the Declaration of Helsinki. Informed consent was obtained from all patients participating in the TOG study. For the GLIOMAT study, informed consent was waived as patients had either previously provided authorization for use of their medical records for research or were deceased.

A total of 300 patients from ten local medical institutions diagnosed with GBM in the period 2006-2017 were included in the study. Inclusion criteria were: pathologically proven GBM according to the 2007 World Health Organization (WHO) Classification of Tumors of the Central Nervous System [23], availability of a pretreatment volumetric contrast enhanced (CE) T1-weighted MRI sequence (slice thickness $\leq 2.00 \mathrm{~mm}$, pixel spacing $1.20 \mathrm{~mm}$, no gap), no substantial imaging artifacts, presence of contrast-enhancing areas, and unifocal lesions. Sequences used were gradient echo using 3D spoiled gradient recalled echo or 3D fast field echo. Availability of the relevant clinical variables was also required: age, treatment scheme, and survival information. A data summary is shown in Table 2. Additional patient data are provided in reference [33].

\subsection{Tumor segmentation and three-dimensional reconstruction}

Active tumor areas are contained between two surfaces shown in Figure 2. The outer, $\Sigma_{E}$ is the interface between the 'normal' tissue and contrast enhancing - macroscopic tumor - areas. The inner, $\Sigma_{I}$, is the interface between the tumor tissue and the necrotic areas. The Digital Imaging and Communications in Medicine 
TABLE 2. Summary of patient data and MRI characteristics.

\begin{tabular}{|lc|}
\hline Patients & \\
\hline Number of patients (censored) & $300(31)$ \\
Age (yr) median [range] & $63[23-86]$ \\
Sex (M, F) & $(134,166)$ \\
Survival (months), median [range] & $14.11,[3.40,96.90]$ \\
\hline MRI characteristics & \\
\hline Pixel spacing (mm), median [range] & $0.89[0.46-1.09]$ \\
Slice thickness (mm), median [range] & $1.50[1.00-4.00]$ \\
Spacing between slices (mm), mean [range] & $1.00[0.50-2.00]$ \\
Field strength (n) & $1.0 \mathrm{~T}(9), 1.5 \mathrm{~T}(264), 3 \mathrm{~T}(27)$ \\
TR ms median [range] & $11.05[4.88-30]$ \\
TE ms median [range] & $3.93[1.48-10.28]$ \\
Flip angle degrees median [range] & $20[8-90]$ \\
Manufacturer (n) & $\mathrm{GE}(142)$, Philips $(98), \operatorname{Siemens}(60)$ \\
\hline
\end{tabular}

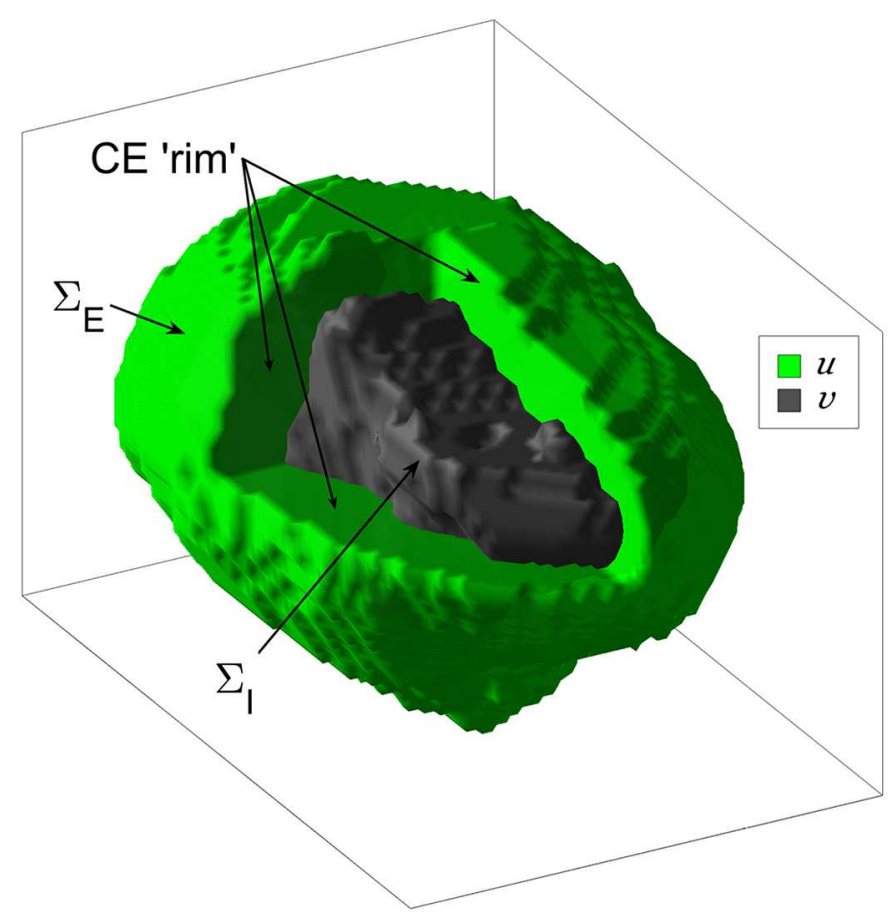

FiguRE 2. An example of the reconstruction of a glioblastoma in 3D from postcontrast T1weighted MRIs. The arrows indicate the regions of necrosis (inner) and contrast enhancement (outer), corresponding to the locations of active tumor cells $(u)$ and necrosis $(v)$ respectively. These regions are used for the computation of the volumes $V_{I}$, enclosed in the $\Sigma_{I}$ surface, and $V_{C E}$, enclosed between $\Sigma_{E}$ and $\Sigma_{I}$.

(DICOM) files were imported into scientific software package Matlab and were preprocessed by using an in-house semiautomatic segmentation procedure. Tumors were automatically delineated by using a gray-level threshold chosen to identify the largest CE tumor volume. Then, the image expert manually corrected each segmentation. The segmentations were supervised by experienced radiologists. A reproducibility study involving three different observed was developed to compare differences between segmentations. The Correlation Coefficient Index 
(CCI) was used to analyze differences between image experts. CCI and $95 \%$ interval confidence were 0.999 and $[0.998,1.000]$ respectively, with $P<0.001$ [31].

Let us denote the total $\left(V_{T}\right)$, and necrotic (or inner) tumor volumes $\left(V_{I}\right)$ as the volumes enclosed by the surfaces $\Sigma_{E}$ and $\Sigma_{I}$ respectively. The CE volume is then defined as $V_{C E}=V_{T}-V_{I}$. These volumes can be easily obtained from the tumor segmentations and the voxel dimensions. Then, the spherical rim width is a rough approximation to the averaged rim width based on the assumption of sphericity of the necrotic and total tumor areas, computed by the equation

$$
\delta_{s}=\left(\frac{3}{4 \pi}\right)^{1 / 3}\left[\left(V_{C E}+V_{I}\right)^{1 / 3}-V_{I}^{1 / 3}\right]
$$

It is possible to define more sophisticated measures of the rim width based on the 3D reconstruction of the tumors and computational geometry approaches as done in [33]. The results were found to be in line with those obtained with the simple measure given by equation (2.3) and thus we will focus here on the spherical rim width.

\subsection{Numerical simulations}

To solve numerically equations (2.1), we used second order finite difference methods for the spatial variable and a modified Euler method for the time variable. Simulations were carried out in two spatial dimensions. The boundary conditions were of Dirichlet type $u( \pm \infty)=0, v( \pm \infty)=0$ with the boundaries placed far enough to avoid disturbance to the tumor bulk dynamics. Initial conditions were taken as given by $u(x, y, 0)=0.2 \operatorname{sech}\left(2 \sqrt{x^{2}+y^{2}}\right), v(x, y, 0)=0$, corresponding a small dysplasia and an exponential infiltration, as it is expected to be the case for Fisher-Kolmogorov type dynamics. A total of 408 numerical simulations were carried out to simulate the tumor growth and the appearance of the necrotic core in the whole parameter range described in Section 2.2.

To calculate the tumor rim width for the virtual tumors $\delta_{s}$ we fixed two threshold values, $K_{u}=0.7$, and $K_{v}=0.4$ for $u$ and $v$ compartments, respectively, assumed to correspond to the contrast-enhancing or necrosis signals in T1w MRIs. The choice of different values for the threshold comes from the fact that cell density is not the quantity measured in postcontrast T1-weighted images but the contrast agent released to the extracellular medium by the unstable vasculature. In the interface between tumor and normal tissue this happens because of the colonization of the normal brain by the infiltrative tumor component and the destruction of the normal - more robust - vasculature. Thus, a high cell density is necessary to break normal vessels, estimated to be around $K_{u}=0.7$. In tumoral tissue, vessels are more unstable since they usually lack pericytes. Thus, there will be gadolinium released to the medium even when density is lower than that for the normal tissue (we chose $K_{v}=0.4$ ). However, since both the tumor-normal tissue and tumor-necrotic tissue interfaces are sharp in relation with the tumor size, the specific choice of $K_{u}$ and $K_{v}$ is not expected to affect substantially the results.

The distance from the center to the points satisfying $u=K_{u}, v=K_{v}$ provided the radius of the regions $\Sigma_{E}$ and $\Sigma_{I}$ and the difference between them was taken to be $\delta_{s}$.

Then, for the full range of values used for the parameters $D, \rho$ and $\beta$, we developed an automatic algorithm to calculate the rim width $\delta_{s}\left(t_{k}\right)$, the tumor radius, $R\left(t_{k}\right)$ and the tumor velocity $v\left(t_{k}\right)$, where $t_{k}$ is a particular (discrete) time in the simulation and the velocity was obtained from sequential changes in the tumor radius, $v\left(t_{k}\right)=\left(R\left(t_{k}\right)-R\left(t_{k-1}\right)\right) /\left(t_{k}-t_{k-1}\right)$. An example is shown in Figure 7 .

\subsubsection{Statistical methods}

The Kolmogorov-Smirnov test [12, 16, 37] was used to analyze the normality of the distributions. For variables with negative results in the test, the Spearman correlation coefficient, $r$, was used to analyze the association between different variables $[4,38]$. The t-Student test was used to quantify the correlation significance, obtaining the $P$-value, $P$. 


\section{THEORETICAL STUDY OF THE MODEL}

\subsection{Qualitative analysis for the model without diffusion}

In order to understand the dynamics of our system let us first study the evolution problem without diffusion, given by the set of ordinary differential equations

$$
\begin{aligned}
& \frac{\mathrm{d} u}{\mathrm{~d} t}=\rho u(1-u-v)-\rho \beta u(u+v-\gamma) \phi, \\
& \frac{\mathrm{d} v}{\mathrm{~d} t}=\rho \beta u(u+v-\gamma) \phi .
\end{aligned}
$$

Tumor growth can be splited in two different dynamical regimes depending on the value of $\phi$, thus we will study both cases. First, when $\phi=0$, i.e., $u(t)+v(t) \leq \gamma$, tumor growth is given by

$$
\frac{\mathrm{d} u}{\mathrm{~d} t}=\rho u(1-u), \quad u(0)=u_{0}
$$

The fixed points of this one-dimensional equation are $u=0$ (unstable) and $u=1$ (stable). It is straightforward to calculate the time that tumor takes to reach the threshold value $\gamma$ :

$$
t_{*}=\frac{1}{\rho} \log \left[\frac{\gamma\left(1-u_{0}\right)}{u_{0}(1-\gamma)}\right]
$$

with $\gamma>u_{0}$.

When the tumor reaches this threshold value, $u(t)+v(t)=\gamma$ and therefore $\phi(u, v)=1$, and then the dynamics is ruled by the following system of differential equations

$$
\begin{aligned}
& \frac{\mathrm{d} u}{\mathrm{~d} t}=\rho u(1-u-v)-\rho \beta u(u+v-\gamma), \quad u\left(t_{*}\right)=\gamma, \\
& \frac{\mathrm{d} v}{\mathrm{~d} t}=\rho \beta u(u+v-\gamma), \quad v\left(t_{*}\right)=0 .
\end{aligned}
$$

Since equations (3.3) are an autonomous planar dynamical system, the possible dynamics in the phase space can be completely understood. We start by noting that the equilibrium points are given by the set of points $M=\left\{\left(0, v_{*}\right), v_{*} \in \mathbb{R}\right\}$. We are interested in values of $v_{*} \geq 0$. Linearizing equations (3.3) around the equilibria $\left(0, v_{*}\right)$ we obtain that the eigenvalues of the Jacobian matrix are given by

$$
\lambda_{1}=0, \quad \lambda_{2}=\rho\left(1-v_{*}-\beta v_{*}+\beta \gamma\right),
$$

with eigenvectors $(0,1)$ and $\left(1, \beta\left(v_{*}-\gamma\right) /\left(1-v_{*}-\beta\left(v_{*}-\gamma\right)\right)\right.$, respectively (we are assuming that $\left.\lambda_{2} \neq 0\right)$. These points are nonhyperbolic. If $v_{*}<(1+\beta \gamma) /(1+\beta)$ then the fixed point $\left(0, v_{*}\right)$ possesses a local unstable manifold and a local center manifold. Otherwise, $\left(v_{*}, 0\right)$ has a local stable manifold and a local center manifold.

A straightforward application of the center manifold theory shows that the center manifold of $M$ is the same set, i.e., $W^{c}(M)=M$. Moreover, the points of $M$ satisfying $v_{*}<(1+\beta \gamma) /(1+\beta)$ are unstable, while the points over the center manifold satisfying $v_{*}>(1+\beta \gamma) /(1+\beta)$ are stable. Moreover, from the biological point of view, we are interested on the points $\left(0, v_{*}\right)$ satisfying $v_{*} \leq 1$

Since the initial conditions for equations (3.3) are $u\left(t_{*}\right)=\gamma$ and $v\left(t_{*}\right)=0$, we are interested in an orbit connecting $(\gamma, 0)$ with $\left(0, v_{*}\right)$, with $v_{*}>(1+\beta \gamma) /(1+\beta)$. To find that orbit, we first rule out the presence of limit cycles using the Dulac's criterion (see for instance [39]). Let $X$ be the first quadrant of the phase 
space $(u, v)$,

$$
X=\{(u, v): u>0, v>0\} .
$$

Let us first prove the following proposition:

Proposition 3.1. There exist no limit cycles of the equations (3.3) in the region $X$.

Proof. We define the auxiliary function $g(u, v)=1 / u$. This function is continuously differentiable on $X$, so it satisfies the requirements of the Dulac's criterion. Then

$$
\frac{\partial}{\partial u}(g \dot{u})+\frac{\partial}{\partial v}(g \dot{v})=-\rho<0,
$$

for all $(u, v) \in X$ and therefore, due to Dulac's criterion, there exists no limit cycle for equations (3.3) in the region $X$.

Now we formulate a proposition on the global stability of the fixed points $\left(0, v_{*}\right)$ :

Proposition 3.2. Let $\left(0, v_{*}\right)$, with $(1+\beta \gamma) /(1+\beta)<v_{*} \leq 1$. Then these equilibrium points are globally stable on the region $S$, where $S$ is the region enclosed between the following sets:

$$
\begin{aligned}
& S_{1}=\{(u, v): u \geq 0, \gamma \leq v \leq 1\} \\
& S_{2}=\{(u, v): u+v \geq \gamma\} \\
& S_{3}=\{(u, v): \gamma \leq u \leq 1, v \geq 0\} \\
& S_{4}=\{(u, v): u+v \leq 1\}
\end{aligned}
$$

Proof. Let us consider the region $S$. Denote by $\vec{n}$ the outward-pointing normal vector for each segment on the boundary of $S$. The flow on each segment of the boundary of the region $S$ is given by

$$
\left.X\right|_{\partial S}=\left.\langle\vec{n},(\rho u(1-u-v)-\rho \beta u(u+v-\gamma), \rho \beta u(u+v-\gamma))\rangle\right|_{\partial S}
$$

where $\langle$,$\rangle denotes the inner product. Thus, we can compute the flow on each of the boundaries of S$ :

- on the segment $\partial S_{1}:=\{u=0, \gamma \leq v \leq 1\}$, we have $\left.X\right|_{\partial S_{1}}=0$;

- on the line $\partial S_{2}:=\{u+v=\gamma\}$, we get $\left.X\right|_{\partial S_{2}}=-\rho u(1-\gamma)<0$;

- on the segment $\partial S_{3}:=\{\gamma \leq u \leq 1, v=0\},\left.X\right|_{\partial S_{3}}=-\rho \beta u(u-\gamma)<0$;

- finally, on the segment $\partial S_{4}:=\{u+v=1\}$, we find that $\left.X\right|_{\partial S_{4}}=0$.

Thus, $S$ is a positive invariant set of equations (3.3) and all trajectories which go into $S$ remain there (see Fig. 3). The fixed points $\left(0, v_{*}\right)$, with $(1+\beta \gamma) /(1+\beta)<v_{*} \leq 1$ are part of $S$. Since by proposition 3.1 there exist no limit cycles of (3.3) in the region $S$, this implies that the fixed points $\left(0, v_{*}\right)$ with $(1+\beta \gamma) /(1+\beta)<v_{*} \leq 1$ are globally asymptotically stable in $S$.

Since the point $(0, \gamma)$ belongs to $S$, there exists an orbit joining it with $\left(0, v_{*}\right)$, for $(1+\beta \gamma) /(1+\beta)<v_{*} \leq 1$. We want to note that the possible dynamics when $\phi=1$ are restricted to the initial conditions for equations (3.3), which are final values obtained when $\phi=0$, so not all possible orbits of the phase portrait are possible.

It is possible to obtain the explicit form of the equation of the orbits of equations (3.3)

$$
\frac{\mathrm{d} u}{\mathrm{~d} v}=\frac{1+\beta \gamma-(1+\beta) u-(1+\beta) v}{\beta(u+v-\gamma)}, \quad u(0)=\gamma,
$$




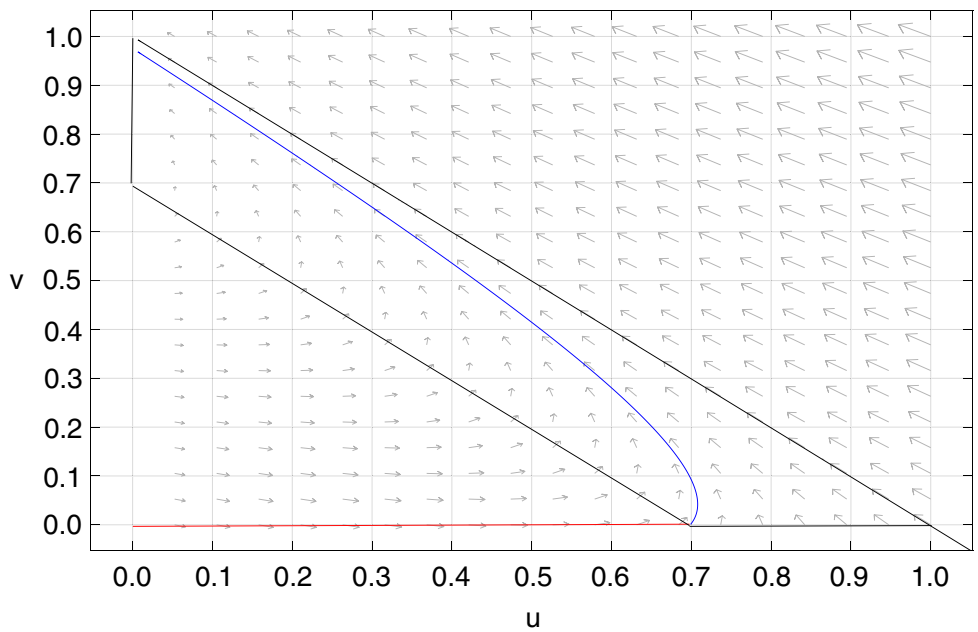

Figure 3. Phase portrait of the dynamical system (3.3) for $\rho=0.05, \beta=1$ and $\gamma=0.7$. The region enclosed by the bold polygon is the positively invariant manifold $S$. The red curve corresponds to the logistic growth for the first stage of the tumor dynamics (Eqs. (2.1) for $\phi=0$ ). The blue curve corresponds to the second stage of the tumor dynamics (Eqs. (2.1), for $\phi=1)$.

whose solution is

$$
u=\frac{1+\beta \gamma}{1+\beta}-v-\frac{\gamma-1}{1+\beta}[\beta+W(z)]
$$

where

$$
z=-\frac{e^{w}}{(\gamma-1)(\beta+1)}
$$

with

$$
w=\frac{\beta+v-\beta \log ((\gamma-1)(\beta+1))+\gamma \beta(\log ((\gamma-1)(\beta+1))-1)}{\beta(\gamma-1)} .
$$

The function $W(z)$ is called the Lambert function [9]. Thus, the solution (3.6) is an orbit connecting the points $(\gamma, 0)$ and $\left(0, v_{*}\right)$ with $(1+\beta \gamma) /(1+\beta)<v_{*} \leq 1$ which is the solution we were looking for (see Fig. 4$)$. Figure 4 shows both dynamics of the tumor growth, i.e., the first dynamics (red curve) corresponds to the case when $u+v \leq \gamma$ and the second one (blue curve) corresponds to the case when $u+v>\gamma$.

Finally, it is interesting to note that for $\gamma=1$, which corresponds to the trajectory of the limit case, $u=1-v$ (see the portrait phase, Fig. 3), we can calculate the explicit solution. Substituting $u=1-v$ in equation (3.3b) we get the differential equation:

$$
\frac{\mathrm{d} v}{\mathrm{~d} t}=\rho \beta(1-\gamma)(1-v), \quad v\left(t_{*}\right)=0,
$$

whose solution is

$$
v=1-e^{-\rho \beta(1-\gamma)\left(t-t_{*}\right)},
$$



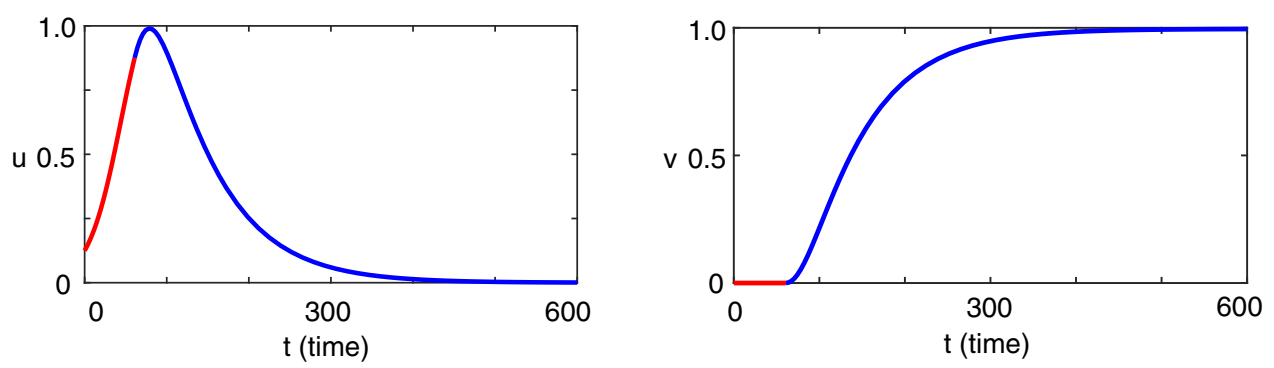

FiguRE 4. Solutions of equations (3.3). The red curve corresponds to the dynamics of the tumor and necrotic compartments for $u+v \leq \gamma$. The blue trajectory corresponds to the dynamics of the tumor and necrotic compartments for $u+v>\gamma$.

and the solution for $u$ is

$$
u=e^{-\rho \beta(1-\gamma)\left(t-t_{*}\right)} .
$$

The solutions for $v$ and $u$ define upper and lower bounds for the solutions of biological interest (see Fig. 3).

\subsection{Stability, phase portrait and traveling waves analysis for the model with diffusion}

Here we consider equations (2.1) in one spatial dimension

$$
\begin{aligned}
& \frac{\partial u}{\partial t}=D \frac{\partial^{2} u}{\partial x^{2}}+\rho u(1-u-v)-\rho \phi(u, v)\left[\beta_{1} u(u-\gamma)+\beta_{2} u v\right] \\
& \frac{\partial v}{\partial t}=\rho \phi(u, v)\left[\beta_{1} u(u-\gamma)+\beta_{2} u v\right] .
\end{aligned}
$$

It is convenient to rescale equations $(2.1)$ by defining

$$
\bar{t}=\rho t, \quad \bar{x}=x\left(\frac{\rho}{D}\right)^{1 / 2},
$$

and, omitting the bars in $\bar{x}$ and $\bar{t}$ for simplicity, we obtain

$$
\begin{aligned}
& \frac{\partial u}{\partial t}=\frac{\partial^{2} u}{\partial x^{2}}+u(1-u-v)-\beta u(u+v-\gamma) \phi, \\
& \frac{\partial v}{\partial t}=\beta u(u+v-\gamma) \phi .
\end{aligned}
$$

\subsubsection{Transition time between dynamical regimes}

We will first consider the case $u(x, t)+v(x, t) \leq \gamma$, i.e., $\phi=0$, and calculate the time it takes for the tumor to reach the threshold value $\gamma$. For the sake of clarity, let us briefly recapitulate the mode of reasoning for this equation (see for instance [28] for a complete exposition).

A traveling wave ansatz $z=x-c t$ turns the Fisher-Kolmogorov equation into a second order ordinary differential equation for $U(z)=u(x, t)$,

$$
U^{\prime \prime}(z)+c U^{\prime}(z)+U(1-U)=0
$$


with $U(-\infty)=1$ and $U(\infty)=0$ and where $c \geq c_{\min }=2$. Taking $\varepsilon=1 / c^{2}$, we can look for asymptotic solutions for $0<\varepsilon \ll 1$. Making the transformation

$$
\xi=\varepsilon^{1 / 2} z, \quad u(z)=g(\xi)
$$

we obtain

$$
\begin{aligned}
& \varepsilon \frac{\mathrm{d}^{2} g}{\mathrm{~d} \xi^{2}}+\frac{\mathrm{d} g}{\mathrm{~d} \xi}+g(1-g)=0, \\
& g(-\infty)=1, g(\infty)=0
\end{aligned}
$$

and further requiring $g(0)=1 / 2$. This equation looks like as a singular perturbation problem for the parameter $\varepsilon$. Thus, looking for solutions of the form

$$
g(\xi)=g_{0}+\varepsilon g_{1}(\xi)+\cdots,
$$

we obtain solutions for $g_{0}, g_{1}$ and so on and in terms of the original variables $U$ and $z$ we get

$$
U(z)=\frac{1}{1+e^{z / c}}+O\left(\frac{1}{c^{2}}\right)
$$

Now, when for the first time $u(x, t)=U(z)=\gamma$, the tumor cells begin to die and, for this case,

$$
z_{*} \simeq c \log \left(\frac{1}{\gamma}-1\right) .
$$

Moreover, we can calculate the value of $t=t_{*}$, where that event occurs,

$$
t_{*} \simeq \frac{x}{c}-c \log \left(\frac{1}{\gamma}-1\right)
$$

which depends on the spatial variable $x$. In particular, we can determine the value of $t_{*}$ for which the tumor cells begin to die just at the maximum value of the tumor, that is, at the center of the tumor, $x=0$ :

$$
t_{*} \simeq-c \log \left(\frac{1}{\gamma}-1\right)
$$

which depends on the speed of the tumor $c$ and the value threshold $\gamma$.

\subsubsection{Wave speed analysis and stability of equilibrium points}

Performing the change of variable $z=x-c t$ to the system (3.12) we obtain the equation

$$
\begin{array}{r}
U^{\prime \prime}+c u^{\prime}+u(1-U-V)-\beta u(U+V-\gamma)=0, \\
c V^{\prime}+\beta U(U+V-\gamma)=0,
\end{array}
$$

where $u(x, t)=U(z)$ and $v(x, t)=V(z)$.

To proceed, we transform the ODEs (3.19) to an autonomous system

$$
U^{\prime}=W
$$




$$
\begin{aligned}
W^{\prime} & =-c W-U(1-U-V)-\beta U(U+V-\gamma), \\
V^{\prime} & =-\frac{\beta}{c} U(U+V-\gamma) .
\end{aligned}
$$

The equilibria of equations (3.20c) are of the form $(U, W, V)=\left(0,0, v_{*}\right), v_{*}$ being an arbitrary real number. From the biological point of view, we are interested in values of $0 \leq v_{*} \leq 1$.

Thus, we have a continuum of equilibrium points on the $v$ axis. If we linearize equations (3.20) around the fixed points $\left(0,0, v_{*}\right)$, we can calculate the eigenvalues of the Jacobian matrix, which are given by

$$
\lambda_{1}=0, \lambda_{2,3}=-\frac{c}{2} \pm \frac{1}{2} \sqrt{c^{2}-4\left[1-v_{*}-v_{*} \beta+\gamma \beta\right]} .
$$

To avoid the fixed points from having a behavior of spiral type (and therefore $U$ to be negative somewhere), the eigenvalues must be all real. This can happen in two ways. First, if $1-v_{*}-v_{*} \beta+\gamma \beta<0$ the root of the expression (3.21) is always positive and therefore $\lambda_{2}$ is positive and $\lambda_{3}$ is negative. Thus, the points which satisfy the relation $v_{*}>(1+\gamma \beta) /(1+\beta)$ are unstable. The second possibility occurs when $1-v_{*}-v_{*} \beta+\gamma \beta>0$ and $c^{2} \geq 4\left[1-v_{*}-v_{*} \beta+\gamma \beta\right]$, then the eigenvalues $\lambda_{2}$ and $\lambda_{3}$ are always negative and an application of the center manifold theory shows that these points $\left(0,0, v_{*}\right)$ with $v_{*}<(1+\gamma \beta) /(1+\beta)$ are stable. Thus, the minimum wave speed $c_{\min }$ of the system is

$$
c_{\min }=2 \sqrt{1-v_{*}-\beta v_{*}+\beta \gamma},
$$

and therefore $c \geq c_{\min }$. In terms of original dimensional system, the range of wave speeds satisfies

$$
c \geq 2 \sqrt{\rho D} \sqrt{1-v_{*}-\beta v_{*}+\beta \gamma} .
$$

This value can therefore be smaller than the Fisher-Kolmogorov limit [28].

The initial conditions for equations (3.20) come from the final values of the initial tumor dynamics (3.13) and are $(U, W, V)=(\gamma,-\gamma(1-\gamma) / c, 0)$. Thus, the desired solution is the one that joins the points $(U, W, V)=$ $(\gamma,-\gamma(1-\gamma) / c, 0)$ with $\left(0,0, v_{*}\right)$, where $v_{*}<(1+\gamma \beta) /(1+\beta)$. Figure 5 shows a concatenation of this orbit

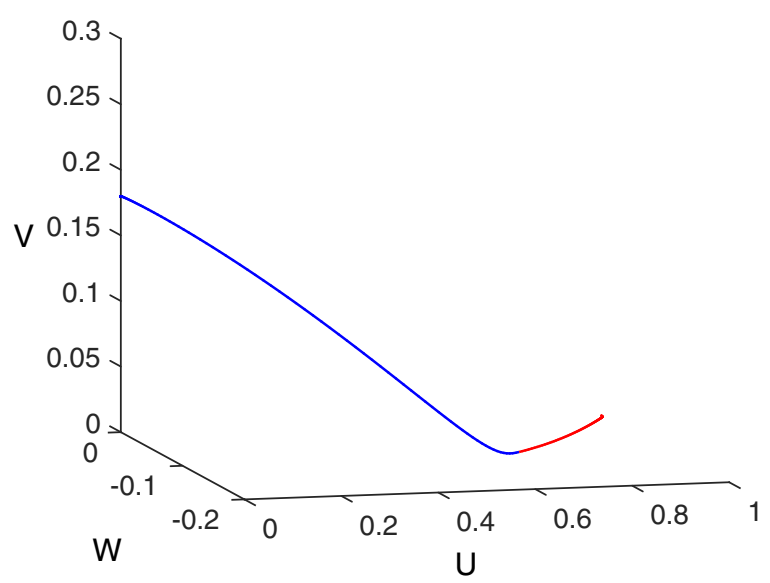

Figure 5. Solution of equations (3.20) (blue line) with initial data $(U, W, V)=(\gamma,-\gamma(1-$ $\gamma) / c, 0)$. This initial condition comes from the final value of the initial tumor dynamics (3.13), whose solution is also represented in this figure (red line). The values of the parameters used in this plot were $\beta=1, \gamma=0.7$ and $c=2$. 
(blue line) together with the orbit (red line) corresponding to the initial dynamics for $U(z)+V(z) \leq \gamma$. As in the case without diffusion, the dynamics of the system with necrosis $(\phi=1)$ is established by the final conditions of the system without necrosis $(\phi=0)$.

In summary, we would like to highlight the results obtained in this section, which are, for the model without diffusion the calculation of equilibrium points and their local and global asymptotic stability and the obtention of analytical solutions for some of the orbits of the model. For the model with diffusion we calculated the transition time between the two dynamic regimes, studied the stability of equilibrium points and constructed explicit solutions for the value of the minimum speed of the traveling wave.

\section{NumericAl RESUlts}

\subsection{The dynamics of equation (2.1) qualitatively resembled the behavior of real GBMs}

We first studied numerically the dynamics of equations (2.1), to test the model's simulations against the observed growth dynamics of real GBMs. Figures 6 and 7 show the typical dynamics observed in simulations of the model. Typical dynamics starting from a localized tumor results in the development of a growing rim of tumor cells surrounding the internal necrotic region. This is also apparent in the cell density profiles depicted in Figure 7a. This dynamics corresponds to a linear growth in tumor radius (see Fig. 7b), an asymptotically constant rim width (Fig. 7c) and a constant tumor growth asymptotic speed (Fig. 7d).

\section{2. 'In silico' simulations show that tumor rim width correlates with growth speed}

We performed a large number of numerical simulations of the model equations (2.1) computing the asymptotic tumor rim widths and growth speeds. We did that for 408 parameter combinations spanning uniformly the full parameter space.

a

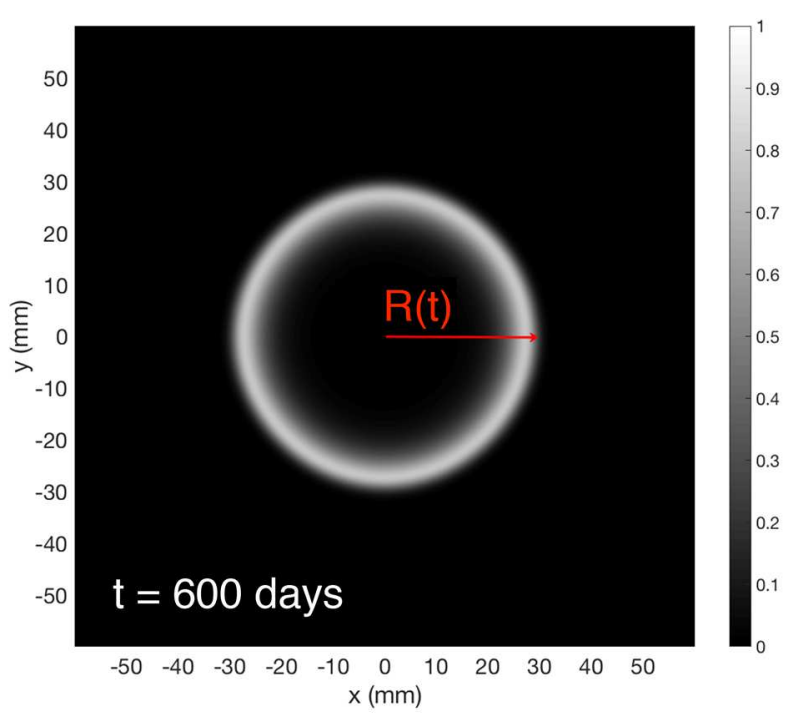

b

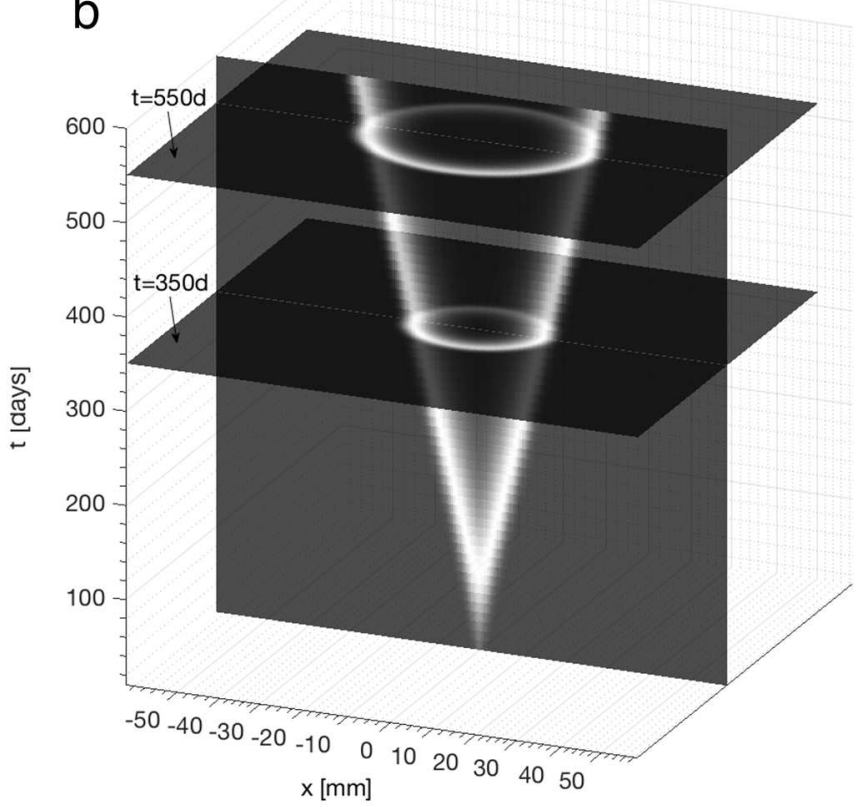

Figure 6. Tumor cell density obtained from equations (2.1) for parameters values $D=0.01$, $\beta=1$ and $\rho=0.06$. (a) Pseudocolor plot of the tumor cell density (in $2 \mathrm{D}$ ) for $t=600$ days.

(b) Three-dimensional reconstruction of the tumor growth dynamics. 

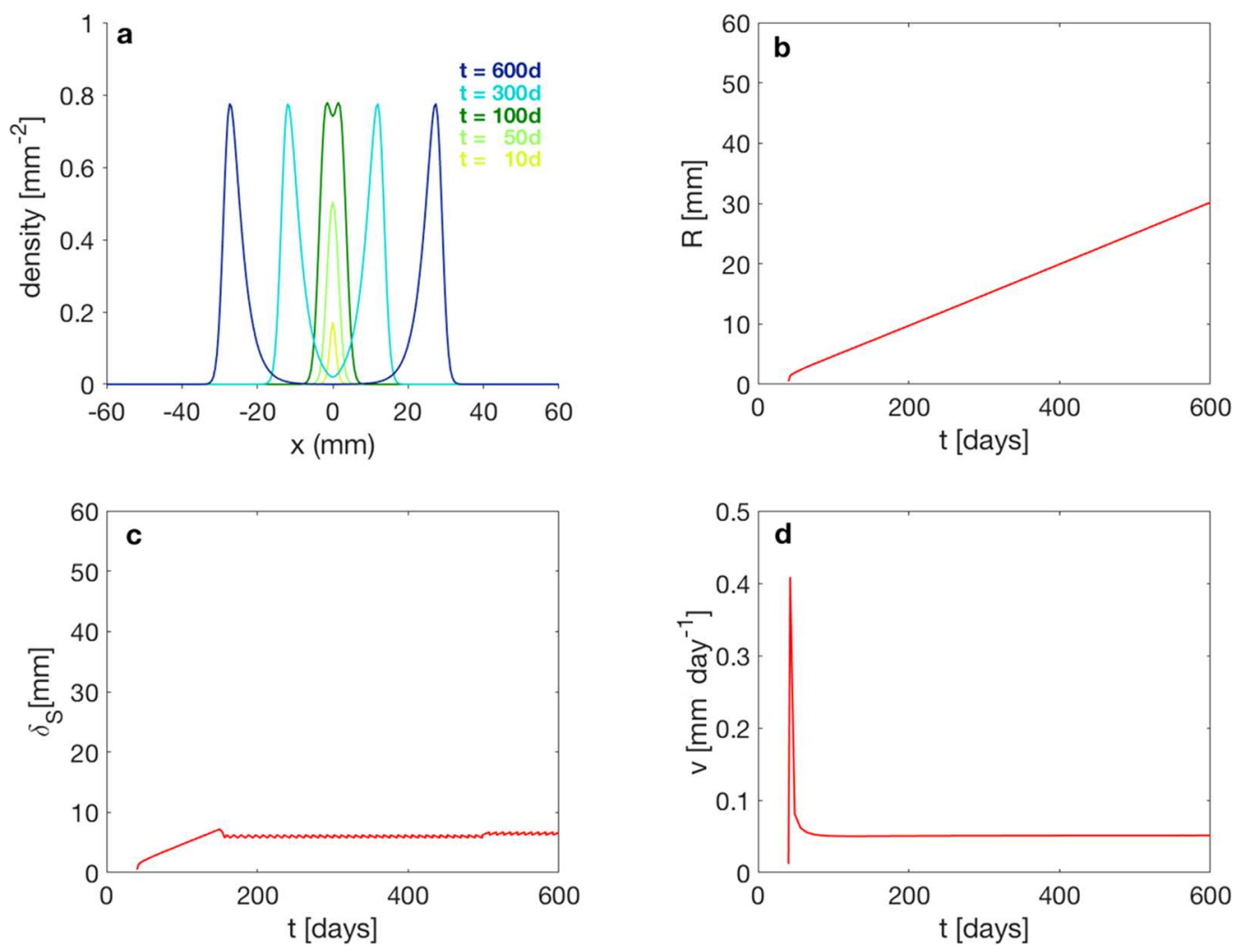

FiguRE 7. Tumor cell dynamics obtained from equations (2.1) for parameters values $D=$ $0.01 \mathrm{~mm}^{2}$ day $^{-1}, \beta=1$ and $\rho=0.06$ day $^{-1}$. (a) Snapshots of tumor cell density $u(x, 0)$ for times $t=10,50,100,300$ and 600 days. (b) Tumor radius $R(t)$ dynamics as a function of time.

(c) Contrast enhancing rim width, $\delta_{s}(t)$. (d) Tumor growth speed $v(t)$.

The distribution of rim widths obtained from our simulations is shown in Figure 8.

To validate the results obtained from simulations, we compared the results with data obtained from measurements from real GBMs. To do so, we used the database described in Section 2.3. The distribution of real widths obtained from the patients MRIs was different from the one obtained by an uniform scanning of the parameter space performed in our set of simulations (see Fig. 8b and a respectively).

This can be due to several reasons but most probably either due to the fact that the range of parameters or the uniform distribution within this parameter did not match those of real solutions. To find a subset of computational solutions closer to the set of patients in our dataset we counted the number of patients in each class in Figure 8a and equal number of parameter values providing computational solutions of the same width. After completing the process for all classes we ended up with a set of 300 parameter combinations (and correspondingly simulations of tumor growth) providing the same distribution of widths as the real one (Fig. 8c). In order to analyze the sensibility of the results, we have carried out a study taking 50 samples of virtual random patients and compared for each sample the relation between speed and rim width as shown in Figure 9, where $r$ is the Spearman correlation coefficient. All the statistical analyzes obtained significant $p$-values $P<10^{-18}$.

If we assume that the more aggressive a tumor is, the faster it grows, then the tumor growth speed and the patients survival could be both correlated with tumor contrast enhancing rim width. For the real patient's dataset we have no sequential measurements and therefore we chose to compare the correlation between rim width and survival (see e.g., Fig. 10a). Our results showed a negative Spearman correlation coefficient, $r=$ -0.1086 , between $\delta_{s}$ and survival $(P=0.06)$. This result was in line with previous evidences that showed that 

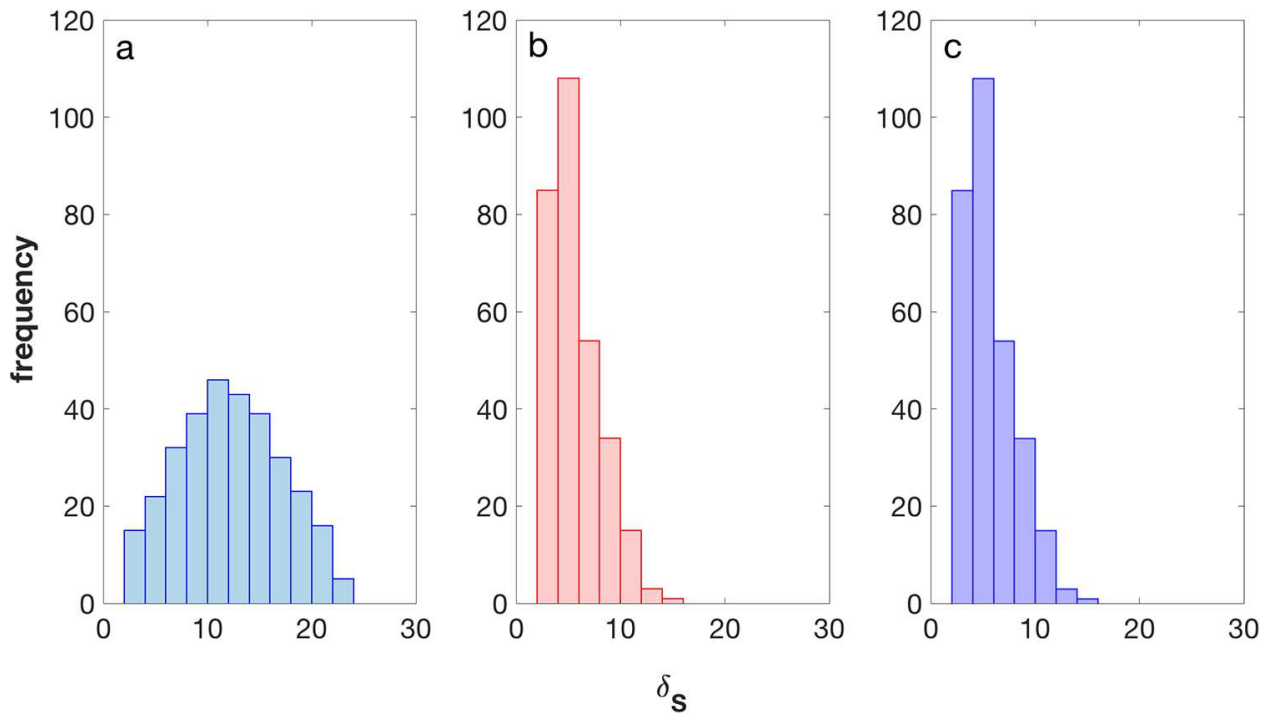

FIgURE 8. Statistical distributions of the rim width $\delta_{S}$ corresponding to: (a) all simulations on a uniform grid in the parameter space. (b) GBM patients database. (c) Virtual distribution constructed from a subset of the virtual patients (see text).

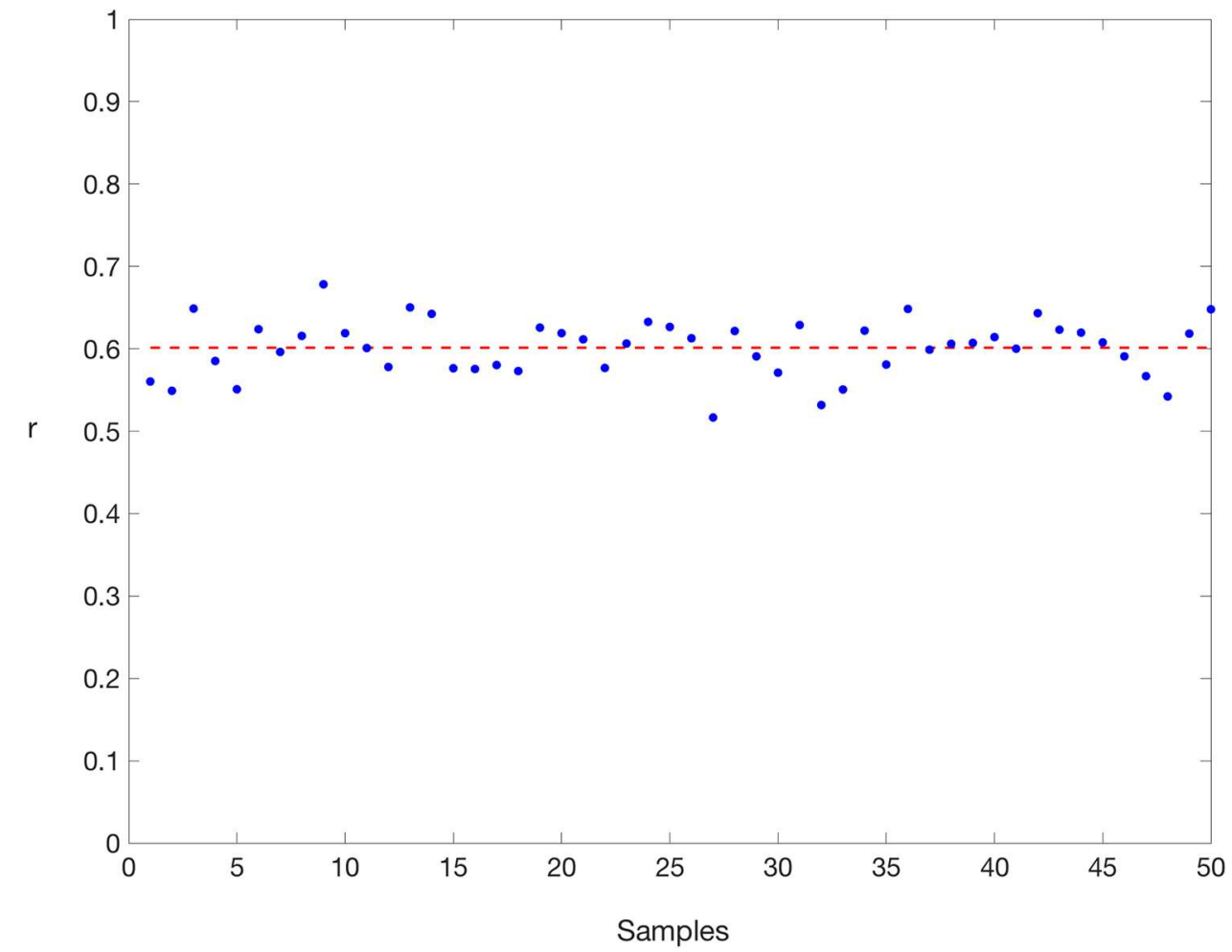

FIGURE 9. Spearman correlation coefficient $r$ between tumor speed and the rim width corresponding to 50 samples of virtual random patients. 

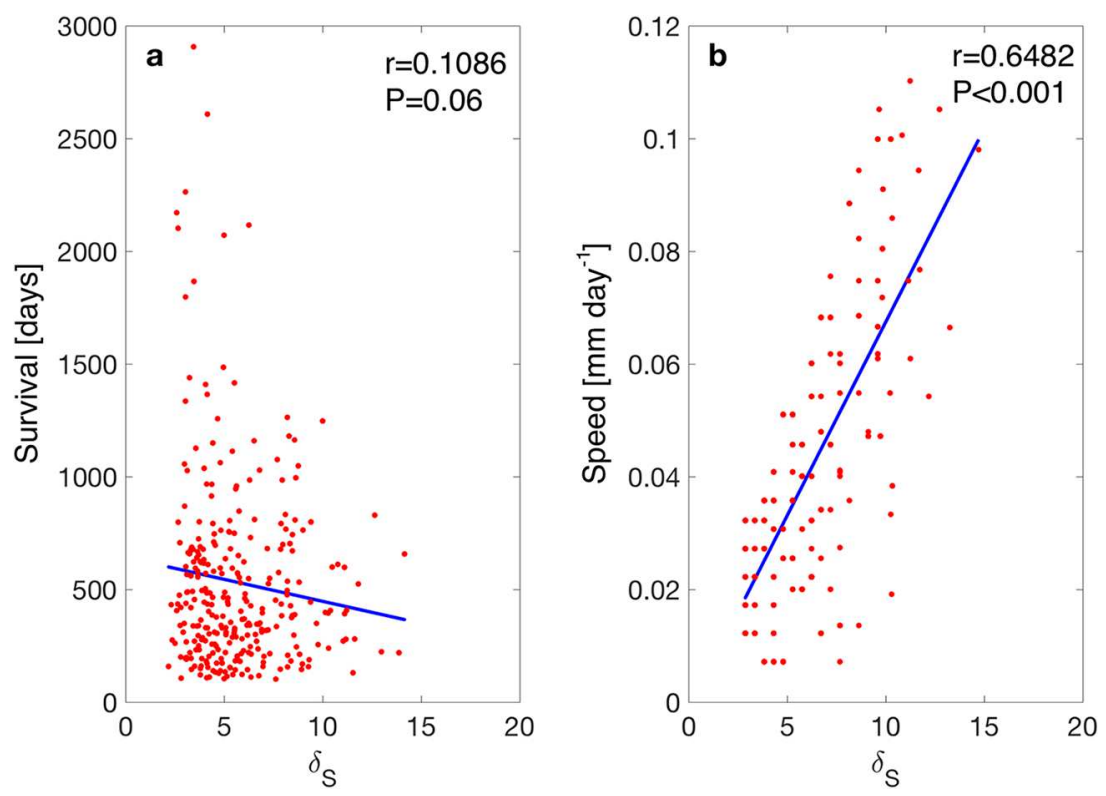

FIgURE 10. Scatter plots and Spearman correlations for: (a) survival versus $\delta_{s}$ from GBM patients data $(r=-0.1086, P=0.06)$, (b) growth speed versus $\delta_{s}$ for virtual patients in the selected virtual GBM subgroup $(r=0.6482, P<0.001)$.

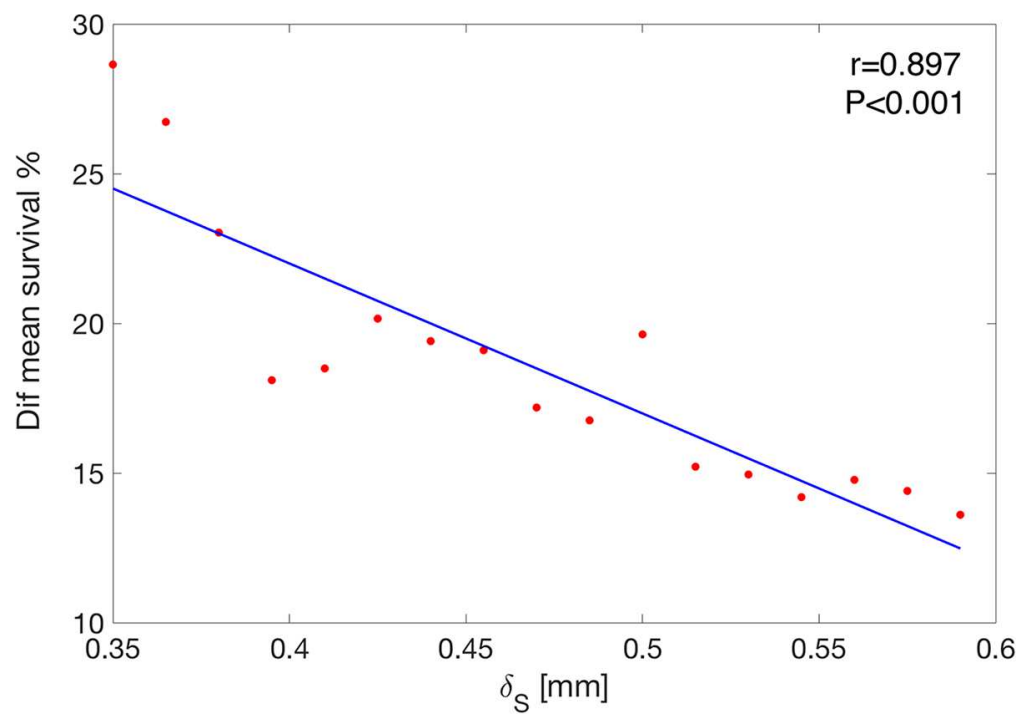

FiguRE 11. Scatter plots and Spearman correlation for the difference in mean survival between subgroups of large versus small $\delta_{s}$ for every threshold splitting the full patient cohort in groups with at least 75 patients $(r=0.897, P<0.001)$. 
large rim widths are associated with worse survival [30, 32, 33] using Kaplan-Meir and other statistical methods. To compare the computational estimates of the rim width with the tumor aggressiveness, we used the tumor growth speed calculated previously in the simulations, obtaining $P<0.001$ and $r=0.6482$. This result is shown in Figure 10b.

We also performed a comparison between the differences in median survival between the groups of patients having rim width smaller and larger than a given $\delta_{s}$ and found a highly significant association with $r=0.897$ and $P<0.001$ (Fig. 11), where we can observe how the differences are higher for smaller $\delta_{s}$ values. Thus, both previous evidences $[30,32,33]$ and the ones obtained in this work point out in the same direction that larger rim widths are indicative of more aggressive tumors.

\section{DisCUSSION AND CONCLUSIONS}

In this paper we wanted to study the connection between the rim width of tumor areas in glioblastoma as observed in postcontrast pretreatment T1-weighted images and tumor aggressiveness. This connection has been observed in survival analyses in studies using large patient datasets $[30,32,33]$. The idea was based on intuitions based on a simple GBM growth mathematical model [35]. However, a more in-depth analysis was in order since the model lacked a correct description of several biological phenomena and no direct comparison between the model predictions and the real patient data had been performed. This was the goal of this paper where we first developed a simple mathematical model of GBM growth including necrosis. The model developed was a minimal one accounting for the presence of tumor an necrotic areas. We first carried out a theoretical study of the model without diffusion, calculating the equilibrium points, its stability and establishing a series of basic properties. For the case of the model with diffusion, we studied the properties of traveling wave solutions. In particular, we obtained an expression for the minimal wave velocity of the travelling wave.

However, the key element of our study was the numerical study of the connection between tumor rim width and aggressiveness, that was quantified through the velocity of the travelling wave obtained from the growth of a localized initial data. The typical tumor growth dynamics was in agreement with the model simulations (see e.g., Fig. 6) and showed that traveling wave appear having a constant width and speed depending on the parameters, as obtained in the theoretical study of the model and simulations (e.g., Fig. 7). The numerical simulations of the model presented here were in line (Fig. 10b) with both the previous results observed clinical evidence $[30,32,33]$ and the new analysis reported here (see e.g., Figs. 10a and 11).

Thus, our results provide theoretical support for the hypothesis that tumor aggresiveness estimates can be obtained from pretreatment postcontrast T1-weighted MRIs of GBM patients. In fact, equation (3.22) provides an analytical estimate for the minimal wave speed that is in line with the Fisher-Kolmogorov velocity but includes a correction term including the model specific parameters $\beta$ and $\gamma$. As a future work, it would be interesting to find explicit formulae for the tumor rim width that could be correlated with the wave speeds to get numeric estimates for the growth speed (or other parameters), from the initial images.

Acknowledgements. We would like to acknowledge J.M. Borrás and M. Claramonte (Neurosurgery Department), C. López, M. Calvo (Radiology Department) and E. Arregui (Radiation Oncology Department) from Hospital General Universitario de Ciudad Real; J.A. Barcia and L. Iglesias (Neurosurgery Department), J. Avecillas (Radiology Department) from H. Clínico San Carlos, Madrid; C. Velásquez and J. Martino (Neurosurgery Department) from Hospital Universitario Marqués de Valdecilla and Fundación Instituto de Investigación Marqués de Valdecilla, Santander; P. C. Lara and R. Cabrera (Radiation Oncology Department) from Hospital Universitario de Gran Canaria Doctor Negrín; B. Asenjo and I. Herruzo (Radiology Department), M. Benavides (Medical Oncology Department) from Hospital Regional Universitario de Málaga; D. Albillo (Radiology Department), M. Navarro, J. M. Villanueva and J. C. Paniagua (Medical Oncology) and L. Pérez-Romasanta (Radiation Oncology Department) from Hospital Clínico Universitario de Salamanca; B. Meléndez (Pathology Department), Á. Rodríguez (Neurosurgery Department) and R. Moreno (Radiology) from Hospital Virgen de la Salud (Toledo); T. Revert (Radiology Department) from Hospital de Manises; A. Ramos (Radiology Department), J. Sepúlveda (Neuro-oncology Department), A. Hernández-Laín (Pathology Department) from Hospital 12 de Octubre (Madrid), and E. Arana (Radiation Oncology Department) from Instituto Valenciano de Oncología, for providing the data 
used in this study and for discussions. This research has been supported by the Ministerio de Economía y Competitividad/FEDER, Spain (grant number MTM2015-71200-R), Junta de Comunidades de Castilla-La Mancha (grant number SBPLY/17/180501/000154) and James S. Mc. Donnell Foundation 21st Century Science Initiative in Mathematical and Complex Systems Approaches for Brain Cancer (Collaborative award 220020560).

\section{REFERENCES}

[1] S. Abrol et al., Radiomic phenotyping in brain cancer to unravel hidden information in medical images. Top. Magn. Reson. Imag. 26 (2017) 43-53.

[2] J.C.L. Alfonso et al., The biology and mathematical modelling of glioma invasion: a review. J. R. Soc. Interface. 14 (2017) 20170490.

[3] A. Alvarez-Arenas, J. Belmonte-Beitia and G.F. Calvo, Nonlinear waves in a simple model of high-grade glioma. Appl. Math. Nonlinear Sci. 1 (2016) 405-422.

[4] D.G. Altman, Practical Statistics for Medical Research, 4th edn. Chapman \& Hall, London (1991).

[5] A. Amelot et al., Surgical decision making from image-based biophysical modeling of glioblastoma: not ready for primetime. Neurosurgery 80 (2017) 793-799.

[6] A. Baldock et al., From patient-specific mathematical neuro-oncology to precision medicine. Front. Oncol. 3 (2013) 62.

[7] A. Baldock et al., Patient-specific metrics of invasiveness reveal significant prognostic benefit of resection in a predictable subset of gliomas. PLoS One 9 (2014) e99057.

[8] P.K. Burgess et al., The interaction of growth rates and diffusion coefficients in a three-dimensional mathematical model of gliomas. J. Neuropathol. 56 (1997) 703-740.

[9] R. Corless, G.H. Gonnet, D.E.G. Hare, D.J. Jeffrey and D.E. Knuth, On the Lambert function. Adv. Comput. Math. 5 (1996) 329-359.

[10] Y. Cui et al., Prognostic imaging biomarkers in glioblastoma: development and independent validation on the basis of multiregion and quantitative analysis of MR images. Radiology 278 (2016) 546-553.

[11] Y. Cui et al., Volume of high-risk intratumoral subregions at multi-parametric MR imaging predicts overall survival and complements molecular analysis of glioblastoma. Eur. Radiol. 27 (2017) 3583-3592.

[12] D.A. Darling, The Kolmogorov-Smirnov, Cramer-von Mises Tests. J. Stat. Model. Anal. 28 (1957) $823-838$.

[13] B.M. Ellingson, Radiogenomics and imaging phenotypes in glioblastoma: novel observations and correlation with molecular characteristics. Curr. Neurol. Neurosci. Rep. 15 (2015) 506.

[14] B.M. Ellingson et al., Emerging techniques and technologies in brain tumor imaging. Neuro. Oncol. 16 (2014) 12-23.

[15] B.M. Ellingson et al., Baseline pretreatment contrast enhancing tumor volume including central necrosis is a prognostic factor in recurrent glioblastoma: evidence from single and multicenter trials. Neuro. Oncol. 19 (2017) 89-98.

[16] D.L. Evans, J.H. Drew and L.M. Leemis, The distribution of the Kolmogorov-Smirnov, Cramer-von mises, and AndersonDarling Test Statistic for exponential populations with estimated parameters. Commun. Stat. Simul. Comput. 3 (2007) 13961421.

[17] R.J. Gillies, P.E. Kinahan and H. Hricak, Radiomics: images are more than pictures, they are data. Radiology. 278 (2016) $563-577$.

[18] M. Ingrisch et al., Radiomic analysis reveals prognostic information in T1-weighted baseline magnetic resonance imaging in patients with glioblastoma. Invest. Radiol. 52 (2017) 360-366.

[19] P. Kickingereder et al., Radiomic profiling of glioblastoma: identifying an imaging predictor of patient survival with improved performance over established clinical and radiologic risk models. Radiology 280 (2016) 880-889.

[20] R.L. Klank, S.S. Rosenfeld and D.J. Odde, A Brownian dynamics tumor progression simulator with application to glioblastoma. Converg. Sci. Phys. Oncol. 4 (2018) 015001.

[21] J. Lao et al., A deep learning-based radiomics model for prediction of survival in glioblastoma multiforme. Sci. Rep. 7 (2017) 10353.

[22] Q. Li et al., A fully-automatic multiparametric radiomics model: towards reproducible and prognostic imaging signature for prediction of overall survival in glioblastoma multiforme. Sci. Rep. 7 (2017) 14331.

[23] D.N. Louis et al., The 2007 WHO classification of tumours of the central nervous system. Acta Neuropathol. 114 (2007) 97-109.

[24] A. Martínez-González, G.F. Calvo, L. Pérez-Romasanta and V.M. Pérez-García, Hypoxic cell waves around necrotic cores in glioblastoma: a mathematical model and its therapeutical implications. Bull. Math. Biol. 74 (2012) 2875-2896.

[25] A. Martínez-González, G.F. Calvo, J.M. Ayuso, I. Ochoa, L.J. Fernández and V.M. Pérez-García, Hypoxia in gliomas: opening therapeutic opportunities using a mathematical-based approach. Adv. Exp. Med. Biol. 936 (2016) 11-29.

[26] D. Molina et al., Geometrical measures obtained from pretreatment postcontrast T1 weighted MRIs predict survival benefits from bevacizumab in glioblastoma patients. PLoS One 11 (2016) e0161484.

[27] D. Molina, L. Vera, J. Pérez-Beteta, E. Arana and V.M. Pérez-García, Survival prediction in glioblastoma: man versus machine. Scientific Report $\mathrm{n}^{\circ} 5982(2019)$.

[28] J. Murray, Mathematical Biology. Springer, Berlin (2003).

[29] S. Narang et al., Radiomics in glioblastoma: current status, challenges and opportunities. Trasl. Cancer Res. 5 (2016) $383-397$. 
[30] J. Pérez-Beteta et al., Glioblastoma: does the pretreatment geometry matter? A postcontrast T1 MRI-based study. Eur. Radiol. 27 (2017) 163-169.

[31] J. Pérez-Beteta et al., Tumor surface regularity at MR imaging predicts survival and response to surgery in patients with glioblastoma. Radiology 288 (2018) 218-225.

[32] J. Pérez-Beteta et al., Morphological MRI-based features and extent of resection predict survival in glioblastoma. Eur. Radiol. 29 (2019) 1968-1977.

[33] J. Pérez-Beteta, A. Mártinez-González and V.M. Pérez-García, A three-dimensional computational analysis of magnetic resonance images characterizes the biological aggressiveness in malignant brain tumours. J. R. Soc. Interface 15 (2018) 20180503.

[34] J. Pérez-Beteta et al., Morphological features on MR images classify multiple glioblastomas in different prognostic groups. Am. J. Radiol. 40 (2019) 634-640.

[35] V.M. Pérez-García, G.F. Calvo, J. Belmonte-Beitia, D. Diego and L. Pérez-Romasanta, Bright solitary waves in malignant gliomas. Phys. Rev. E 84 (2011) 021921.

[36] M. Protopapa et al., Clinical implications of in silico mathematical modeling for glioblastoma: a critical review. J. Neurooncol. 136 (2018) 1-11.

[37] N.M. Razali and Y.B. Wah, Power comparisons of Shapiro-Wilk, Kolmogorov-Smirnov, Lilliefors and Anderson-Darling tests. J. Stat. Model. Anal. 2 (2011) 21-33.

[38] P. Sprent and N.C. Smeeton, Applied Nonparametric Statistical Methods. Chapman \& Hall, London (2007).

[39] S. Strogatz, Nonlinear Dynamics and Chaos: Studies in Nonlinearity. CRC Press, Boca Raton (2007).

[40] K.R. Swanson, R.C. Rostomily and E.C. Alvord Jr., A mathematical modelling tool for predicting survival of individual patients following resection of glioblastoma: a proof of principle. Br. J. Cancer $\mathbf{9 8}$ (2007) 113-119.

[41] V. Verma et al., The rise of radiomics and implications for oncologic management. J. Natl. Cancer Inst. 109 (2017).

[42] C.H. Wang et al., Prognostic significance of growth kinetics in newly diagnosed glioblastomas revealed by combining serial imaging with a novel biomathematical model. Cancer Res. 69 (2009) 9133-9140.

[43] P. Wangaryattawanich et al., Multicenter imaging outcomes study of the cancer genome atlas glioblastoma patient cohort: imaging predictors of overall and progression-free survival. Neuro. Oncol. 17 (2015) 1525-1537.

[44] M. Zhou et al., Identifying spatial imaging biomarkers of glioblastoma multiforme for survival group prediction. J. Magn. Reson. Imag. 46 (2017) 115-123. 